\title{
Mindfulness-based interventions in secure settings: challenges and opportunities ${ }^{\dagger}$
}

\author{
Dumindu Witharana \& Gwen Adshead
}

\begin{abstract}
SUMMARY
Psychological treatments in secure settings have traditionally been based on psychodynamic and cognitive-behavioural approaches. Recent developments, supported by an emerging evidence base, have generated a significant amount of interest in mindfulness-based psychological therapies and their utility in diverse areas of mental healthcare. In this article we analyse the current evidence base and describe possible mechanisms of action of mindfulness-based psychological approaches. On the basis of the evidence, we advocate a cautious but positive approach to using mindfulness-based interventions in secure services.
\end{abstract}

\section{DECLARATION OF INTEREST}

None.

The most up-to-date statistics from the Ministry of Justice estimate that the number of people detained in forensic services in the UK has increased year on year for a decade, rising from 2750 in 1998 to nearly 4000 by December 2008 (Ministry of Justice 2010). These statistics further show that 1501 people were admitted to secure hospitals in England and Wales in 2008, around 60\% of whom were transferred from prisons. These offender patients require treatments for their complex and often treatment-resistant conditions.

More than three-quarters of people treated in secure hospitals are diagnosed with at least one mental illness, and one study suggests that 90\% of the prison population have a mental disorder (Rutherford 2007). Most forensic patients have dual and even triple diagnoses; it is not unusual for an offender patient to have a psychotic illness, a personality disorder and a history of substance misuse and addiction. Affective disorders and common comorbid conditions such as borderline personality disorder are probably underdiagnosed, which may contribute to the high suicide rates in prisons and secure services (13 times higher than in the general population).
These figures demonstrate the extent of the problem, and the need for effective, evidencebased treatment in forensic psychiatric and prison settings. Historically, psychological therapies have played a relatively minor role in the life of offenders and forensic patients. However, the more recently expanded evidence base for psychological therapies (Roth 2006), and National Institute for Health and Clinical Excellence (NICE) guidance on psychological therapy for a variety of disorders, support the development of expanded and comprehensive psychotherapy services in forensic psychiatry. In this article, we review the evidence for mindfulness-based therapies in forensic practice.

\section{Mindfulness-based therapies: what they are and how they work}

\section{'Third-wave' therapies}

In the past 10-15 years, the development of acceptance and mindfulness-based treatments ${ }^{\ddagger}$ has spearheaded a new approach to psychotherapies within the cognitive-behavioural tradition (Ost 2008; Williams 2008a). These so-called thirdwave approaches represent a shift of paradigm in the cognitive-behavioural framework, expanding the field in ways that lead to:

- less emphasis on first-order change (changing the content of negative cognitions)

- a more contextualised approach to clinical phenomena

- a focus on experiential and indirect strategies for change, in addition to direct strategies

- a broader focus of change: changing the emotional context within which negative cognitions function, rather than merely changing the contents of the negative emotions; this is called second-order change (Hayes 2004).

A common feature of the third wave is the emphasis on mindfulness, radical acceptance and spirituality. Aside from differences in the philosophical foundation, the critical difference
ARTICLE

Dumindu Witharana is a consultant forensic psychiatrist with Priory Healthcare, UK, and an honorary clinical senior lecturer at St George's, University of London. He has completed an MA in Mindfulness-Based Cognitive Therapy at the University of Oxford. Gwen Adshead trained as a forensic psychiatrist and group analyst and is now a consultant forensic psychotherapist at Broadmoor Hospital, Crowthorne, UK. Correspondence Dr Dumindu Witharana, Priory Hospital, Longcroft Lane, Felden HP3 OBN UK.Email: duminduwitharana@ priorygroup.com

${ }^{\ddagger}$ For an introduction to this topic in Advances, see Mace C (2007) Mindfulness in psychotherapy: an introduction, 13: 147-154. Ed. 
§Readers may also be interested in the Advances article by Fonagy $\mathrm{P}$, Adshead G (2012) How mentalisation changes the mind, 18: 353-362 Ed. between cognitive-behavioural therapy (CBT) and third-wave psychotherapies is that CBT techniques are primarily antecedent-emotion focused (modifying initial feelings by changing the situation or the cognitions of the situation), whereas mindfulness approaches are primarily responsefocused (modifying behaviour once emotions are experienced) (Hofmann 2008). The techniques of CBT promote adaptive antecedent-focused emotional regulation strategies by focusing on reappraisal of the emotional stimuli. In contrast, mindfulness-based therapies target maladaptive response-focused strategies by discouraging emotional submission (Hofmann 2008).

Most scholars agree that mindfulness practice has its roots in Buddhism (Ludwig 2008), a philosophical tradition that originated in India some 2600 years ago. In Buddhism, mindfulness is considered a vehicle for alleviating suffering, cultivating compassion and attaining wisdom. The traditions of Buddhism postulate that, without being aware of oneself and one's surroundings, one cannot understand the natural laws of life.

Contemporary psychologists have described mindfulness as 'the awareness that arises from paying attention on purpose, in the present moment, non-judgementally, to things as they are' (Williams 2007: p. 47). They further expand the concept as not paying more attention, but paying attention 'wisely'. Ludwig \& Kabat-Zinn (2008) describe mindfulness as 'attending to relevant aspects of experience in a nonjudgmental manner' which would 'foster clear thinking and openheartedness'. Accordingly, the goal of mindfulness is to 'maintain awareness moment by moment, disengaging oneself from strong attachment to beliefs, thoughts or emotions thereby developing a greater sense of emotional well-being'.

A key feature of psychological and psychotherapeutic accounts of mindfulness is the assumption that distress and psychopathology are associated with mental activity that is either hypoactive, hyperactive or stuck in an unhelpful pattern of responding. The mind is 'doing' something that is unhealthy, and it needs to stop 'doing' and pay more attention to 'being'. Mindfulness-based therapies can promote and support the 'being' mode. Williams (2008b) illustrates six distinctive differences between the doing and being modes of mind (Table 1).

\section{Mindfulness and mentalisation}

Mentalisation-based treatment $(\mathrm{MBT})^{\S}$ is another therapy that has been increasingly used to good effect in forensic settings during the past few years. Mentalising (Fonagy 2002) and mindfulness are, to a degree, overlapping constructs in their openness to examining mental states and reflecting without acting. Both involve directing one's attention to one's own experience as a way of mitigating tendencies towards impulsivity and reactivity. Mindfulness and mentalisation equally emphasise the integration of cognitive and affective aspects of mental states in encouraging simultaneous recognition and participation in internal experience. Choi-Kain \& Gunderson (2008) identify three distinctions between the two concepts, in terms of interactions, temporal focus

\section{TABLE 1 The 'doing' and 'being' modes of the mind}

\begin{tabular}{|c|c|c|}
\hline Parameter & Doing mode & Being mode \\
\hline Striving v. non-striving & $\begin{array}{l}\text { Monitoring and striving to close the gap between ideas of } \\
\text { where we are now and where we want to be } \\
\text { Monitoring and striving to keep as wide as possible the } \\
\text { gap between ideas of where we fear we might end up if } \\
\text { we do nothing }\end{array}$ & $\begin{array}{l}\text { Mindfulness focuses on letting go of striving towards or } \\
\text { away from such ideas }\end{array}$ \\
\hline Avoidance $v$. approach & $\begin{array}{l}\text { Causes particular problems when it is motivated by } \\
\text { avoidance of subjective experience }\end{array}$ & $\begin{array}{l}\text { Encourages remaining open, 'turning towards' the difficult } \\
\text { and the unpleasant }\end{array}$ \\
\hline $\begin{array}{l}\text { Thoughts as real } v \text {. thoughts as mental } \\
\text { events }\end{array}$ & $\begin{array}{l}\text { Uses ideas (thoughts and images) as its currency, taking } \\
\text { such thoughts literally }\end{array}$ & $\begin{array}{l}\text { Mindfulness views thoughts as merely thoughts, as } \\
\text { 'products' of the mind that arise, stay around for a while, } \\
\text { then disperse }\end{array}$ \\
\hline $\begin{array}{l}\text { Living in the past and future } v \text {. living in } \\
\text { the present }\end{array}$ & $\begin{array}{l}\text { Solves problems by switching between memories of the } \\
\text { past and anticipation of the future }\end{array}$ & $\begin{array}{l}\text { Focuses on present-moment experience; memories are } \\
\text { recognised as memories that are arising now; future } \\
\text { images are seen for what they are, images using here } \\
\text { and now }\end{array}$ \\
\hline $\begin{array}{l}\text { Indirect (conceptual) experience } v \text {. } \\
\text { direct (non-conceptual) experience }\end{array}$ & $\begin{array}{l}\text { Concerned with manipulating ideas, so the objective } \\
\text { experience is thinking about things; it is conceptual, } \\
\text { language-based, verbal and analytic }\end{array}$ & $\begin{array}{l}\text { The focus is on direct, sensory experience; it is non- } \\
\text { conceptual, intuitive, and experiential }\end{array}$ \\
\hline Automatic $v$. intentional & $\begin{array}{l}\text { Relies on habitual, over-learnt routines that run off } \\
\text { automatically }\end{array}$ & $\begin{array}{l}\text { Involves intentionally paying attention to aspects of the } \\
\text { self and the world }\end{array}$ \\
\hline
\end{tabular}

Source: Williams 2008b, reproduced with permission. 


TABLE 2
\begin{tabular}{|ll|}
\multicolumn{2}{|c|}{ Key distinctions between mindfulness and } \\
mentalisation
\end{tabular}
\begin{tabular}{|ll|}
\hline Mindfulness & Mentalisation \\
\hline $\begin{array}{l}\text { Focus on one's experience } \\
\text { interacting with people and } \\
\text { with inanimate objects }\end{array}$ & $\begin{array}{l}\text { Focus on one's experience } \\
\text { interacting with people }\end{array}$ \\
\hline $\begin{array}{l}\text { Oriented towards present } \\
\text { experience }\end{array}$ & $\begin{array}{l}\text { Oriented towards the past } \\
\text { and future as well as the } \\
\text { present }\end{array}$ \\
\hline $\begin{array}{l}\text { Aims at acceptance of } \\
\text { internal experiences }\end{array}$ & $\begin{array}{l}\text { Emphasises the construction } \\
\text { of representation and } \\
\text { meaning related to internal } \\
\text { experiences }\end{array}$ \\
\hline
\end{tabular}

After Choi-Kain \& Gunderson (2008)

and relationship to internal experiences, and these are listed in Table 2.

\section{Therapeutic actions of mindfulness}

The early therapeutic use of mindfulness techniques for psychopathology was in teaching mindfulness-based stress reduction (MBSR) to general medical patients with chronic stress due to physical health conditions that were considered untreatable (Kabat-Zinn 1990). Early studies suggested that patients who practised regularly experienced reduced pain and stress from their conditions; even though they were still ill, they managed their distress better. Similarly, Marsha Linehan's (1993) programme of dialectical behaviour therapy (DBT) uses mindfulness-based techniques to improve affect regulation in those who seem to struggle with their negative affects, and to reduce their tendency to act impulsively when experiencing strong negative emotions (Robins 2001; Lynch 2007).

Since this early work, there has been extensive study of mindfulness-based interventions applied to different types of psychopathology and practised in many types of clinical mental health setting. The most recent and most compelling evidence relates to the use of mindfulness-based cognitive therapy (MBCT) for the treatment of chronic depression, and this now features in national guidelines for the treatment of depression (National Institute for Health and Clinical Excellence 2009).

\section{Psychological actions}

Mace (2008) describes three therapeutic actions resulting from mindfulness practice that may be influential in achieving beneficial outcomes for patients (Box 1).

Dechaining Dechaining entails the close objective observation of psychological events that the 'bare attention' brings. Dechaining explains a mindfulness practitioner's ability to experience an event in full detail, in a slowed down way, but also how each event is seen to be less firmly linked to things that precede and follow it than ordinary perception suggests. Developing this quality has been found to be important in reducing ruminations and in breaking the cycle of anxiety response typically found in behavioural disorders.

Resensing In resensing, the individual learns to relate to exposure to situations and feelings in a different manner and to welcome experiences as they are attended to, with acceptance and equanimity and without fear and aversion. The freshness of the experience here reflects the absence of old reactions of distress or fear, a feature that is important in treating anxiety and phobic disorders.

Decentring Mindfulness approaches emphasise the adverse effects of believing that one's thoughts necessarily reflect reality and of reacting to them accordingly. Decentring is the capacity to observe one's thoughts and feelings as temporary mental phenomena rather than reflections of the self that are necessarily true. Decentring includes taking a non-judgemental, here-and-now approach towards thoughts and feelings and accepting them as they are. This seems to form the basis of the effectiveness of mindfulness-based approaches in dealing with problematic thoughts such as obsessional or depressive ruminations.

\section{Neurological actions}

At a neurobiological level, certain consistent findings have been found to be associated with the practice of mindfulness:

- increased activation of the dorsolateral prefrontal cortex, an area associated with executive decision-making and attention (Baerentsen 2001);

- increased activity in the anterior cingulate cortex, which plays a role in integration of attention, motivation and motor control;

B0X 1 Therapeutic actions resulting from mindfulness practice

Dechaining Objective observation of psychological events, to break the cycle of ruminations and anxiety responses

Resensing The capacity to accept experiences with equanimity and without fear or aversion

Decentring The capacity to observe one's thoughts and feelings as temporary mental phenomena, rather than necessarily true reflections of reality

(Mace 2008) 
- increased activity in the insula, a region of the brain associated with interoception and processing transient bodily sensations; insular activity has been observed during induction of sad mood (Liotti 2002), experience of pain or disgust (Wright 2004) and the internal generation of emotions (Reiman 1997) as well as guilt (Shin 2000);

- increased grey matter density in the right anterior insula, hippocampus and left temporal gyrus in mindfulness meditators (Holzel 2007).

The most recent findings suggest that MBCT may be helpful in reducing relapse in depression by reducing the kindling tendency of neurons involved in affective responses (Farb 2010; Kuyken 2010). Reduction in firing and kindling rates may lessen overgeneralisation of autobiographical memory that influences mood regulation (Williams 2010).

\section{Application to secure settings}

Mindfulness has achieved good success rates with adult, child and adolescent, and older adult age groups and with people with intellectual disability (Didonna 2009). As far back as 1997, over 240 hospitals and clinics internationally were offering mindfulness-based stress reduction training (Salmon 2009). Dialectical behaviour therapy has a growing evidence base for impulsive and suicidal behaviours, and there is a growing body of research to support the effectiveness of mindfulness for conditions such as recurrent depression, stress and psychosomatic disorders (Singh 2008; Dimidjian 2010).

In contrast, there has been a lack of evidencebased psychological therapies for prisoners and forensic patients until comparatively recently. This is due to a number of factors. First is the uncertainty about whether outcomes should focus on individuals feeling better or behaving better. Second is the complexity of treatment research in (a) samples that are biased by the criminal justice and mental health admission systems, and (b) samples in which most participants have at least two conditions, both of which are severe and treatment resistant.

Secure psychiatric services have tended to follow the prison service in offering psychological and psychoeducational programmes for offending behaviour such as violence or sexual offences. Such interventions may be group or individual. Some psychological interventions are individually tailored to focus on a patient's index offence, especially if it does not fall neatly into the offending programmes that exist. More recently, programmes have been developed that emphasise recovery and social inclusion as therapeutic goals for offenders (Dorkins 2011). These psychodynamic interventions (either group or individual) address relational experiences with others (including the therapist) and/or address early attachment experiences in relation to the offence and behaviour in the service.

Forensic services do not always offer patients diagnosis-specific programmes of psychological intervention, even where there are national guidelines to support them. For example, forensic patients with a diagnosis of schizophrenia will automatically be prescribed medication, but they are not automatically prescribed the 20 or more sessions of cognitive therapy, or the creative therapies, recommended by NICE (National Institute for Health and Clinical Excellence 2009). Depression is not always routinely screened for in forensic settings, despite the high suicide rates in custodial services.

The literature suggests that mindfulness-based therapies are particularly effective for the following conditions:

- unstable negative affect and impulsivity

- affective disorders and depression

- general stress reactions, especially those with a psychosomatic component.

We review below the evidence that these conditions are found in forensic populations.

\section{Unstable negative affect and impulsivity}

Negative affects include anger, rage, pain, panic, disgust, shame and hatred. Early childhood attachments are thought to influence the development of negative affect regulatory systems in the human brain (Schore 2001). A key aspect of affect regulation is the capacity to experience a feeling without acting on it and to reflect on that experience as it is occurring. Those who cannot do this tend to act as soon as they have a thought or feeling (usually negative), and this significantly increases the risk of boundary- and criminal rule-breaking. Impulsivity is influenced by both temperament and genes, and some studies suggest that those who are impulsive have low levels of serotonin (Schweighofer 2008).

A combination of negative affect dysregulation and impulsivity will put a person at high risk of 'acting out' their feelings. The most common types of acting out in this context are behaviours that later affect states and also put the self at risk in some way: substance misuse, self-cutting and overdosing. These behaviours are especially common in mood disorders, and are characteristic of disorders of mood regulation such as borderline 
personality disorder. A subgroup of those who harm themselves also act out against other people, impulsively hitting or attacking. Such behaviours are, of course, more likely when an individual is disinhibited by alcohol or illicit drugs, especially those that increase central monoamine levels.

Severe negative affect dysregulation and impulsivity are difficult to treat with cognitive approaches because patients cannot 'hang on to' cognitions and/or affects long enough to reflect on them and consider alternatives. Wright et al (2009) discuss the difficulty of choosing an alternative conscious response to a trigger stimulus when the individual has limited self-awareness. It can be hard to use cognitive-behavioural interventions to activate an alternative behaviour or thought when the individual reacts too quickly in an emotionally charged situation. Goleman (1995) refers to this phenomenon as the hijacking of the cognitive system by the emotional system. Mentalisationbased therapies have had some success in this area because they increase self-reflective function (Levy 2008), which allows people to pause before they act.

Mindfulness-based approaches such as DBT have been shown to be successful in reducing the frequency of impulsive self-harm and antisocial behaviour. A number of mechanisms of action have been proposed to explain this, and we consider some of them (Box 2) here.

\section{Increased tolerance and capacity to cope}

Mindfulness may help to reduce the emotional reactivity typically elicited by anger-provoking stimuli via a process of sustained, non-judgemental observation of anger-related sensations, without attempts to escape, act on or avoid them (Wright 2009). Thus, it is expected that mindfulness will improve an individual's ability to tolerate anger states and increase their capacity to cope with them effectively. This would lead to extinction of responses and behaviours previously elicited by anger-provoking stimuli.

\section{Separation of thoughts from reality}

Increased mindfulness leads to thoughts being appraised as thoughts and not necessarily as reality. This realisation enables individuals to be less inclined to react to the thinking process, and emotional experience may be less likely to be refired by associated appraisals. When mindfulness is practised regularly, it has been postulated that experience in general is more likely to be regarded without harsh judgement (Harned 2006), thus allowing individuals to view situations from a different viewpoint and respond accordingly.
BOX 2 How mindfulness might reduce impulsivity and antisocial behaviour

- Increasing the individual's tolerance of angry feelings and capacity to cope with them

- Enabling the individual to differentiate between thoughts and reality

- Short-circuiting ruminative thought processes that maintain anger

- Teaching the individual to use the direct experience of the senses in self-monitoring and reality-checking

\section{Short-circuiting ruminative thought processes}

There is considerable evidence that rumination exacerbates negative affects, especially anger, and that it may be a key maintaining factor in chronic anger (Rusting 1998; Borders 2009). Work by Teasdale (1999) on the ways that mindfulness training might interrupt ruminative thought processes suggests a possible indication for mindfulness in this area.

\section{'Being': a focus on direct experience of the senses}

The 'being' mode (Table 1) focuses on the direct experiences of the senses, which informs selfmonitoring and reality-testing (Williams 2008b). Enhanced self-monitoring can enable individuals to make more informed choices and regulate their feelings in response to environmental cues, which in turn will influence their behavioural choices (Novaco 2007; Wright 2009). It can also help them to appraise and respond wisely to potential danger cues and to overcome some of the problems posed by automaticity. According to Brown $\&$ Ryan (2003), those who have high mindfulness skills demonstrate significantly greater self-regulation in emotion and behaviour (Box 3).

BOX 3 Case vignette: controlling self-harm

Ms $X$ is a 24-year-old woman admitted to a medium secure unit from a prison. She has engaged in frequent self-harm for the past 8 years. In the medium secure unit, she participated in an 8-week mindfulness-based therapy programme specifically tailored to address her self-harming behaviour. By the end of the programme Ms X had developed the ability to be more aware of her thoughts and emotions and to appraise thoughts as mental events that pass through the mind rather than as facts or parts of her identity. There was a shift in Ms $X$ 's tendency to react automatically to minor frustrations of life with anger and self-blame. With regular practice of mindfulness skills she became able to take steps to manage her frustrations without resorting to self-harm. 


\section{Clinical depression, psychological distress and self-harm}

Prisons and secure psychiatric residential settings are psychologically demanding environments that require people to draw on a range of internal and external resources in order to survive - resources that many will lack, given the level of psychiatric and psychological morbidity in this population (Singleton 1998). In prisons, there are high rates of both self-harm and suicide (Shaw 2004): 22459 incidents of self-harm were recorded among women in UK prisons in 2007 (Short 2009). Men are equally at risk. Fazel et al (2005) noted a rise in completed suicide in prison between 1978 and 2003. However, with new measures in place to reduce suicides in prisons, this trend appears to have reversed by 2008 (Brooker 2010).

A large proportion of prisoners experience a high level of psychological distress (Harvey 2007): in one study, $65 \%$ of women and $52 \%$ of men obtained scores indicative of distress on the 12-item General Health Questionnaire (HM Inspectorate of Prisons 2007). Prisoners experience isolation, shame and social exclusion, as well as psychiatric diagnoses such as depression and borderline personality disorder, all of which are risk factors for both self-harm and suicide. In addition, the index offence itself may be a 'disaster' for the prisoner/patient, causing both acute and long-term stress. This is particularly the case for family violence and homicide.

A survey of suicides in UK high secure hospitals spanning 29 years (1972 to 2000) found increased rates of suicide among in-patients compared with the general population (Jones 2011). More interestingly, it found that women in high security were more than twice as likely to complete suicide than men, which is a significant deviation from the trend seen in general population. In a survey of suicides in medium secure units in Britain, James (1996) recorded that 9 out of the 19 units that responded reported a death by suicide. James noted that patients in medium secure units often have multiple risk factors for suicide although, in fact, completed suicide in medium security is rare. It is reasonable to infer that, thanks to close observation and stringent patient management techniques in secure hospitals, far more suicides are prevented than actually succeed.

This evidence from prisons and secure psychiatric services clearly suggests that there is a significant subgroup of individuals who are suffering distress, may have clinical depression and may be at enhanced risk of suicide. The therapeutic effectiveness of mindfulness practice for affective disorders has been well demonstrated and an evidence base is firmly established (National Institute for Health and Clinical Excellence 2009). For example, MBCT is effective for chronic depression, especially in reducing the risk of relapse. Mindfulness practice has been shown to increase positive affect and reduce stress reactivity, producing demonstrable effects on brain function. One study reported significant leftward shift in resting electroencephalogram (EEG) patterns of healthy people after 8 weeks of mindfulness practice, which persisted at 4-month follow-up (Davidson 2003). Similar changes in brain activity, together with reduced distress, were recorded in individuals with a history of suicidal depression who completed an 8-week course of MBCT (Barnhofer 2007).

\section{Stress reactions with a psychosomatic component}

There have been few studies of stress in forensic populations, although by inference from prison data, it seems reasonable to assume that a substantial number of forensic patients experience stress on a daily basis. Having a personality disorder or a psychotic disorder reduces the capacity to manage stress, and the personality disorder diagnosis in particular is associated with psychosomatic dysfunction and medically unexplained symptoms (Crimlisk 1998). Mindfulness-based techniques might therefore be of use in this group of forensic patients. Mindfulness training could also form part of healthy living programmes addressing patients' physical health by making them more aware of their bodies.

\section{Other possible indications for mindfulness practice in forensic settings}

In addition to the conditions described above, it is theoretically possible that mindfulness-based therapies might be effective in people who lack compassion for themselves or others, as developing compassion is a key goal of mindfulness practice. Mindfulness practice has an effect on midline prefrontal regions of the brain, which have been implicated in controlling aspects of empathy and insight (Beer 2006; Heisel 2006) and moral reasoning (Anderson 1999; Bechara 2000; Moll 2002; Greene 2004). Furthermore, there is evidence to suggest that conscientiousness - one of the positive 'Big Five' personality traits ${ }^{\text {II }}$ - is correlated with trait mindfulness (Giluk 2009) and, in theory, could therefore be improved by mindfulness practice. (Trait mindfulness is when mindfulness is measured as a personality attribute - a trait - that varies from person to person). However, it is complicated to study the therapeutic 
efficacy of new modes of treatment in groups that are often reluctant to engage in therapy or are contemptuous of attempts to help (McGauley 2003). It is also not certain that profoundly antisocial offenders lack empathy; rather, they might prefer not to use it.

For some offenders, incarceration in secure services is painfully lengthy. An average stay for a patient in a UK high secure hospital is about 8 years. A more palliative, long-term psychological input may be necessary to alleviate the suffering of these individuals (McGauley 2003). Mindfulnessbased interventions in their current forms are usually brief, time-limited and delivered to groups. A possible solution to cater for long-term patients would be to offer identified groups of patients a standard brief course, followed by less frequent maintenance therapy sessions for the duration of the patient's stay in hospital.

Mindfulness practice could also be useful for staff, who have to manage their own negative affects while working with challenging and frightening patients (Lowdell 2009). Staff working in secure settings identify the threat of violence by inmates as their most frequent source of stress. This stress is often exacerbated by the challenges of dealing with offenders who denigrate or attack those who give them care. Staff may label the symptoms of mental illness as 'bad behaviour' and resort to retaliating with punitive measures (Cohen 2003). Mindfulness-based stress reduction has been used in other healthcare professional groups to good effect (Shapiro 2007), and could therefore theoretically play a role in helping forensic staff manage stress and reduce burnout (Box 4).

\section{Challenges to the use of mindfulness in forensic settings}

There are a number of challenges to the use of mindfulness in forensic setting, some relating to its theory, others to its practice.

\section{Concept and criterion validity}

Theoretical challenges include ongoing confusion about the definition and conceptualisation of mindfulness, especially in research. There are debates as to whether mindfulness has been sufficiently operationalised to make research possible (Bishop 2002; Baer 2003; Coffey 2010). Some have questioned the external and internal criterion validity of mindfulness research altogether (Singh 2008). No one has carried out a proper trial involving forensic patients, not least because it has not yet been possible to show definitively that they have specific mindfulness deficits.
BOX 4 Case vignette: stress reduction for healthcare staff

Ms T works as a nurse manager in a very demanding low secure ward. Most of the patients in the ward have a personality disorder in addition to a mental illness. The ward is frequently understaffed because of high staff sickness rates. Ms T very often feels mentally and physically exhausted after a work shift. She recently attended an 8-week MBCT programme aimed at staff. After completing the course she started using the mindfulness technique of three-minute breathing spaces just before starting the shift, during the lunch break and at the end of a shift. She finds regular use of the technique extremely helpful in keeping her emotions under control and helping her to make rational decisions. The practice has improved her work performance and she feels less psychologically exhausted after a shift.

\section{Risk of adverse effects}

One of the practical challenges is the possibility that mindfulness techniques might have a negative effect, making patients worse (Mace 2008). This is a particular concern with forensic patients, who are likely to have severe psychiatric illness. Both mentally ill and healthy people have reported exacerbated restlessness, anxiety, depression, guilt and hallucinosis during meditation training retreats (Albeniz 2000). Experienced meditators have reported restlessness, self-criticism and selfdoubt during early phases of their meditation training (Mace 2008). In our experience, negative side-effects such as anxiety, depression and psychotic breakdown can occur in forensic patients during psychodynamic psychotherapy; hence, extensive exploratory psychotherapy is often not used in this group of patients. The possibility of generating distressing and underresearched sideeffects in patients raises concerns, particularly in a medico-legally sensitive secure therapeutic environment.

\section{Cultural acceptability}

Another practical challenge may be the cultural acceptability of mindfulness-based interventions to patients, carers and professionals in Western mental health systems (Howells 2010). Until 1520 years ago, the word 'meditation' was considered by many to be suspect and associated with images of fraudulent mysticism (Kabat-Zinn 1990); and this, albeit to a lesser degree, may still remain the case in some quarters. Mindfulness is taught and practised in the Western world independently of the rich religious and cultural tradition of its origins (Linehan 1993). Howells et al (2010) alerts 
us to the dangers of severing mindfulness from its roots and treating it in relative isolation from the deeper philosophical and psychological system that generated the concept. Whether mindfulness in isolation of the Buddhist cultural tradition would deliver expected outcomes, beyond a narrow therapeutic remit, remains to be seen.

Qualities such as inwardness, reflection and introspection (which are core aspects of mindfulness) are not widely valued or practised in Western societies, which emphasise action and striving for objects or goals. This might be particularly true in forensic settings, where psychological practice is underpinned by punishment-oriented values. Whether a more extensive and widespread use of mindfulnessbased interventions that primarily focus on compassion and acceptance beyond the narrow remits of DBT would create a greater resistance in forensic settings remains to be seen.

\section{Conclusions}

A number of evidence-based indicators suggest that mindfulness-based therapies would and could make a useful contribution to the treatment of conditions that afflict mentally disordered offenders. Hence, we recommend a cautious, but positive approach towards using mindfulnessbased interventions in secure settings. Such an approach would increase the range of treatments available, and in the long term would provide a valuable evidence base for a rigorous therapeutic outcome evaluation. Howells et al (2010) identify three areas of clinical and criminogenic needs (factors shown to predict future offending) that are potentially remediable through mindfulness training: poor affect regulation, poor anger control and impulsivity.

In our view, mindfulness-based interventions contain unique features - such as the ability to target the 'emotional brain' rather than the 'cognitive brain', a transdiagnostic reach, a nonjudgemental and compassionate base, and an approach that sees the individual as a 'companion in a journey' rather than a 'patient to be explored' - that could prove crucial in engaging and helping a very challenging patient group.

\section{References}

Albeniz A, Holmes J (2000) Meditation: concepts, effects and uses in therapy. International Journal of Psychotherapy 5: 49-58.

Anderson SW, Bechara A, Damasio H, et al (1999) Impairment of social and moral behavior related to early damage in human prefrontal cortex. Nature Neuroscience 2: 1032-7.

Baer RA (2003) Mindfulness training as a clinical intervention: a conceptual and empirical review. Clinical Psychology: Science and Practice 10: 125-43.
Baerentsen KB (2001) Onset of meditation explored with fMRI. Neuroimage 13: S297.

Barnhofer T, Duggan D, Crane C, et al (2007) Effects of meditation on frontal [alpha]-asymmetry in previously suicidal individuals. NeuroReport 18: $709-12$.

Bechara A, Damasio H, Damasio AR (2000) Emotion, decision making and the orbitofrontal cortex. Cerebral Cortex 10: 295-307.

Beer JS, John OP, Donatella S, et al (2006) Orbitofrontal cortex and social behavior: integrating self-monitoring and emotion-cognition interactions. Journal of Cognitive Neuroscience 18: 871-9.

Bishop SR (2002) What do we really know about mindfulness-based stress reduction? Psychosomatic Medicine 64: 71-84.

Borders A, Earleywine M, Jajodia A (2009) Could mindfulness decrease anger, hostility, and aggression by decreasing rumination? Aggressive Behavior 36: 28-44.

Brooker C, Flynn J, Fox C (2010) Trends in self-inflicted deaths in prisons in England and Wales (2001-2008): towards targeted interventions. Journal of Aggression, Conflict and Peace Research 2 (4): 34-43.

Brown KW, Ryan RM (2003) The benefits of being present: mindfulness and its role in psychological well-being. Journal of Personality and Social Psychology 84: 822-48.

Choi-Kain LW, Gunderson JG (2008) Mentalization: ontogeny, assessment, and application in the treatment of borderline personality disorder. American Journal of Psychiatry 165: 1127-35.

Coffey KA, Hartman M, Fredrickson BL (2010) Deconstructing mindfulness and constructing mental health: understanding mindfulness and its mechanisms of action. Mindfulness 1: 235-53.

Cohen F (2003) Mental illness, cruel and unusual punishment, and dignitarian values. Correctional Mental Health Report 5: 33-48.

Crimlisk HL, Bhatia K, Cope H, et al (1998) Slater revisited: 6 year follow up study of patients with medically unexplained motor symptoms. BMJ 316: 582-6.

Davidson RJ, Kabat-Zinn J, Schumacher J, et al (2003) Alterations in brain and immune function produced by mindfulness meditation. Psychosomatic Medicine 65: 564-70.

Didonna F (ed) (2009) Clinical Handbook of Mindfulness. Springer.

Dimidjian S, Kleiber BV, Segal ZV (2010) Mindfulness-based cognitive therapy. In Cognitive and Behavioral Theories in Clinical Practice (eds N Kazantzis, MA Reinecke, A Freeman): 307-31. Guilford Press.

Dorkins E, Adshead G (2011) Working with offenders: challenges to the recovery agenda. Advances in Psychiatric Treatment 17: 178-87.

Farb NAS, Anderson AK, Mayberg H, et al (2010) Minding one's emotions: mindfulness training alters the neural expression of sadness. Emotion 10: 25-33.

Fazel S, Benning R, Danesh J (2005) Suicide in male prisoners in England and Wales, 1978-2003. Lancet 366: 1301-2.

Fonagy P, Gergely G, Jurist E, et al (2002) Affect Regulation, Mentalization and the Development of the Self. Other Press.

Giluk TL (2009) Mindfulness, Big Five personality, and affect: a metaanalysis. Personality and Individual Differences 47: 805-11.

Goleman D (1995) Emotional Intelligence. Bantam Press

Greene JD, Nystrom LE, Engell AD, et al (2004) The neural bases of cognitive conflict and control in moral judgment. Neuron 44: 389-400.

Harned MS, Banawan SF, Lynch TR (2006) Dialectical behavior therapy: an emotion focused treatment for borderline personality disorder. Journal of Contemporary Psychotherapy 36: 67-75.

Harvey J (2007) Young Men in Prison: Surviving and Adapting to Life Inside. Willan Publishing.

Hayes SC (2004) Acceptance and commitment therapy and the new behavior therapies: mindfulness, acceptance and relationship. In Mindfulness and Acceptance: Expanding the Cognitive-Behavioral Tradition (eds SC Hayes, CM Follette, MM Linehan): 1-29. Guilford Press. 
Heisel AD, Beatty MJ (2006) Are cognitive representations of friends' request refusals implemented in the orbitofrontal and dorsolateral prefrontal cortices? A cognitive neuroscience approach to 'theory of mind' in relationships. Journal of Social and Personal Relationships 23: 249-65.

HM Inspectorate of Prisons (2007) The Mental Health of Prisoners: A Thematic Review of the Care and Support of Prisoners with Mental Health Needs. HM Inspectorate of Prisons.

Hofmann SG, Asmundson GJG (2008) Acceptance and mindfulnessbased therapy: new wave or old hat? Clinical Psychology Review 28: $1-16$

Hofmann SG, Sawyer AT, Witt AA, et al (2010) The effect of mindfulnessbased therapy on anxiety and depression: a meta-analytic review. Journal of Consulting and Clinical Psychology 78: 169-83.

Holzel BK, Ott U, Hempel H, et al (2007) Differential engagement of anterior cingulated and adjacent medial frontal cortex in adept meditators and non-meditators. Neuroscience Letters 421: 16-21.

Howells K, Tennant A, Day A, et al (2010) Mindfulness in forensic mental health: does it have a role? Mindfulness 1: 4-9.

James A (1996) Suicide reduction in medium security. Journal of Forensic Psychiatry 7: 406-12.

Jones RM, Hales H, Butwell M, et al (2011) Suicide in high security hospital patients. Social Psychiatry and Psychiatric Epidemiology 46: 723-31.

Kabat-Zinn J (1990) Full Catastrophe Living: Using the Wisdom of Your Body and Mind to Face Stress, Pain and IIIness. Delcorte.

Kuyken W, Watkins E, Holden E, et al (2010) How does mindfulnessbased cognitive therapy work? Behaviour Research and Therapy 48: $1105-12$.

Levy KN (2008) Psychotherapies and lasting change. American Journal of Psychiatry 165: 556-9.

Linehan MM (1993) Cognitive-Behavioral Treatment of Borderline Personality Disorder. Guilford Press.

Liotti M, Mayberg HS, McGinnis S, et al (2002) Unmasking diseasespecific cerebral blood flow abnormalities: mood challenge in patients with remitted unipolar depression. American Journal of Psychiatry 159: 1830-40.

Lowdell A, Adshead G (2009) The best defence: institutional defence against anxiety in forensic services. In Therapeutic Relationships with Offenders (eds A Aiyeghusi, J Clarke-Moore): 53-69. Jessica Kingsley.

Ludwig DS, Kabat-Zinn J (2008) Mindfulness in medicine. JAMA 300: 1350-2.

Lynch TR, Trost WT, Salsman N, et al (2007) Dialectical behavior therapy for borderline personality disorder. Annual Review of Clinical Psychology 3: 181-205.

Mace C (2008) Mindfulness and Mental Health: Therapy, Theory and Science. Routledge.

McGauley G. Humphrey M (2003) Contribution of forensic psychotherapy to the care of forensic patients. Advances in Psychiatric Treatment 9: $117-24$

Ministry of Justice (2010) Statistics of Mentally Disordered Offenders 2008 England and Wales (Ministry of Justice Statistics Bulletin). Ministry of Justice.

Moll J, de Oliveira-Souza R, Eslinger PJ, et al (2002) The neural correlates of moral sensitivity: a functional magnetic resonance imaging investigation of basic and moral emotions. Journal of Neuroscience 22: 2730-6.

National Institute for Health and Clinical Excellence (2009) Depression: The Treatment and Management of Depression in Adults (Update) (Clinical Guideline 90). NICE (http://guidance.nice.org.uk/CG90).

Novaco RW (2007) Anger dysregulation. In Anger, Aggression and Interventions for Interpersonal Violence (eds TA Cavell, KT Malcolm): 3-54. Lawrence Erlbaum.
Ost L-G (2008) Efficacy of the third wave of behavioral therapies: a systematic review and meta-analysis. Behaviour Research and Therapy 46: 296-321.

Reiman EM, Lane RD, Ahren GL, et al (1997) Neuroanatomical correlates of externally and internally generated human emotions. American Journal of Psychiatry 154: 918-25.

Robins CJ, Ivanoff AM, Linehan M (2001) Dialectical behavior therapy. In Handbook of Personality Disorder: Theory, Research and Treatment (ed WJ Livesley): 437-59. Guilford Press.

Roth A, Fonagy P (2006) What Works for Whom? A Critical Review of Psychotherapy Research. Guilford Press.

Rusting C, Nolen-Hoeksma S (1998) Regulating responses to anger: effects of rumination and distraction on angry mood. Journal of Personality and Social Psychology 74: 790-803.

Rutherford M, Duggan S (2007) Forensic Mental Health Services: Facts and Figures on Current Provision. Sainsbury Centre for Mental Health.

Salmon P, Santorelli S, Kabat-Zinn J (2009) Intervention elements promoting high adherence to mindfulness-based stress reduction (MBSR) programs in the clinical behavioral medicine setting. In The Handbook of Health Behavior Change (3rd edn) (eds SA Shumaker, JK Ockene, KA Riekert): 271-86. Springer Publishing.

Schore AN (2001) Effects of a secure attachment relationship on right brain development, affect regulation, and infant mental health. Infant Mental Health Journal 22: 7-48.

Schweighofer N, Bertin M, Shishida K, et al (2008) Low serotonin levels increase delayed reward discounting in humans. Journal of Neuroscience 28: 4528-32.

Shapiro SL, Brown KW, Biegel GM (2007) Teaching self-care to caregivers: effects of mindfulness-based stress reduction on the mental health of therapists in training. Training and Education in Professional Psychology 1: 105-15.

Shaw J, Baker D, Hunt IM, et al (2004) Suicide by prisoners: national clinical survey. British Journal of Psychiatry 184: 263-7.

Shin LM, Dougherty DD, Orr SP, et al (2000) Activation of anterior paralimbic structures during guilt-related script-driven imagery. Biological Psychiatry 48: 43-50.

Short V, Cooper J, Shaw J, et al (2009) Custody vs care: attitudes of prison staff to self-harm in women prisoners: a qualitative study. Journal of Forensic Psychiatry and Psychology 20: 408-26.

Singh NN, Lancioni GE, Wahler RG, et al (2008) Mindfulness approaches in cognitive behavior therapy. Behavioral and Cognitive Psychotherapy 36: $1-8$.

Singleton N, Meltzer H, Gatward R (1998) Psychiatric Morbidity Among Prisoners. Office for National Statistics.

Teasdale JD (1999) Metacognition, mindfulness and the modification of mood disorders. Clinical Psychology and Psychotherapy 6: 146-55.

Williams JMG (2008a) Experimental psychopathology and psychological treatment. In Inside Psychology: A Science over 50 Years (ed P Rabbitt): 281-90. Oxford University Press.

Williams JMG (2008b) Mindfulness, depression and modes of mind. Cognitive Therapy and Research 32: 721-33.

Williams JMG (2010) Mindfulness and psychological process. Emotion 10: $1-7$.

Williams M, Teasdale J, Segal Z (2007) The Mindful Way through Depression: Freeing Yourself from Chronic Unhappiness. Guilford Press.

Wright P, He G, Shapira NA, et al (2004) Disgust and the insula: fMRI responses to pictures of mutilation and contamination. NeuroReport 15: 2347-51

Wright S, Day A, Howells K (2009) Mindfulness and the treatment of anger problems. Aggression and Violent Behavior 14: 396-401.

\section{MCO answers}

1 e $\quad 2$ e $\quad 3 d \quad 4$ c $\quad 5 d$ 


\section{MCOs}

Select the single best option for each question stem

1 'Third-wave' psychotherapeutic approaches include:

a cognitive-behavioural therapy

b psychodynamic psychotherapy

c behavioural therapy

d Gestalt psychology

e mindfulness-based cognitive therapy.

\section{Mindfulness meditation produces the} following change in the brain:

a shrinking of the right cerebral cortex

$\mathrm{b}$ increased volume of the third ventricle

c a spike and wave pattern on EEG

$d$ reduced blood flow to the brain stem

$\mathrm{e}$ increased activity of the insular region.
3 Mindfulness-based interventions have not been used in managing:

a chronic depression

b stress

c eating disorders

d motor neuron disease

e suicide and self-harm.

4 Which of the following is not a challenge against using mindfulness-based interventions in secure settings?

a possible side-effects

b lack of an established evidence base on effectiveness

c high rates of suicide and self-harm in forensic and prison populations

d operationalisation of mindfulness e unfavourable attitudes towards qualities such as inwardness, reflection and introspection in Western societies.

5 Which of the following is not true?

a most forensic patients have from more than one psychiatric diagnosis

b some studies suggest that $90 \%$ of the prison population have a mental disorder

c suicide rates in prisons are significantly higher than in the general population

d prisons are environments of low psychological distress

e personality disorders are significantly associated with psychosomatic illnesses. 